\title{
Spontaneous Formation of Micrometer-Size Inorganic Peapods
}

\author{
Soumyajit Roy,* Maria T. Rijneveld-Ockers, Jan Groenewold, Bonny W. M. Kuipers, \\ Hans Meeldijk, and Willem K. Kegel* \\ Van't Hoff Laboratory for Physical and Colloid Chemistry, Debye Institute, University of Utrecht, \\ Padualaan 8, 3584 CH Utrecht, The Netherlands
}

\author{
Received February 8, 2007
}

\begin{abstract}
We show that polyoxometalate (ammonium phosphomolybdate) Keggin in aqueous dispersions upon sonication spontaneously transforms into micrometer-sized, peapod-shaped structures. The formation of these peapods is preceded by the generation of spherical aggregates. The particles have been characterized experimentally by time-resolved dynamic light scattering (DLS), transmission electron microscopy (TEM), and scanning TEM with a high-angle annular dark field detector (STEM-HAADF) for energy-dispersive X-ray (STEM/EDX) elemental analyses. A pathway for the phenomenon is proposed.
\end{abstract}

\section{Introduction}

Inorganic metal oxide-based clusters, polyoxometalates or POMs, have been used extensively as model systems to study transformations in molecular architecture. ${ }^{1}$ Recent work has demonstrated that complex and large single-molecule POM clusters may even spontaneously form superstructure of colloidal size (i.e., on the order of $10-100 \mathrm{~nm}^{2-4}$ ). Here we address the following question: can very simple sparingly soluble salts of polyoxometalates, such as the ammonium salt of phosphododecamolybdate Keggin, ${ }^{5}$ also show comparable superstructure formation? Indications come from observations that dilute solutions of this Keggin salt tend to scatter light, ${ }^{6}$ pointing to the presence of objects on colloidal length scales. Recently, phosphododecatungstate Keggin has been used in combination with AOT microemulsions or as templates to generate fibrous, star-like, and other interesting architectures on colloidal length scales. $^{7}$

Even as early as the 1930s, complex structure formation with phophatide coacervates had been observed. ${ }^{8}$ Interestingly, though it is known that the ammonium salt of phosphododecamolybdate Keggin forms a colloidal dispersion in water, ${ }^{6}$ the characteristics of the particles of this dispersion have not been investigated until now. However, a lot of fundamental work has been done with the ammonium phosphomolybdate Keggin. For instance, spectroscopic investigations on the formation of the phosphomolybdate Keggin in aqueous and organic solvents, ${ }^{9}$ a DFT study of their stability, ${ }^{10}$ a quantum mechanical study of their thermal

* To whom correspondence should be addressed. (S.R.) E-mail: s.roy@chem.uu.nl.Fax: (+31)30-253-3870.(W.K.K.)E-mail: w.k.kegel@ chem.uu.nl.

(1) (a) Pope, M. Heteropoly and Isopoly Oxometalates; Springer: Berlin, 1983 (b) Hill, C., Ed. Chem. Rev. Thematic Issue on Polyoxometalates 1998, 98.

(2) Liu, T. J. Am. Chem. Soc. 2002, 124, 10942-10943.

(3) Liu, T.; Diemann, E.; Li, H.; Dress, A. W. M.; Müller, A. Nature 2003, $426,59-62$

(4) Zhu, Y.; Cammers-Goodwin, A.; Zhao, B.; Dozier, A.; Dickey, E. Chem.Eur. J. 2004, 10, 2421-2427.

(5) (a) Keggin, J. F. Nature 1933, 131, 908-909. (b) Keggin, J. F. Proc. R. Soc. London, Ser. A 1934, 144, 75-100.

(6) Vogel, A. I. Qualitative Inorganic Analysis; Longman Scientific and Technical: Essex, England, 1987.

(7) (a) Li, M.; Mann, S. Langmuir 2000, 16, 7088-7094. (b) Rautaray, D.; Sainkar, S. R.; Sastry, M. Langmuir 2003, 19, 10095-10099. (c) Mandal, S.; Rautaray, D.; Sastry, M. J. Mater. Chem. 2003, 13, 3002-3005. (d) For a very recent report on Keggin POM microtube formation, see Xin, Z.; Peng, J.; Wang, T.; Xue, B.; Li, L.; Wang, E. Inorg. Chem. 2006, 45, 8856-8858.

(8) For an overview, see Bungenberg de Jong, H. G. In Colloid Science;Kruyt, H. R., Ed.; Elsevier, Amsterdam, 1949; Vol. II, pp 477-482.

(9) Ueda, T., Toya, T., Hojo, M. Inorg. Chim. Acta. 2004, 357, 59-65. decomposition, ${ }^{11}$ and studies of their catalytic activity ${ }^{12-14}$ are only a few. Extensive investigations have also been carried out to explore the nature of related POMs in solution ${ }^{15-21}$ and can be traced back to 1783,22 yet interestingly, the nature of colloidal objects in an aqueous dispersion of the ammonium phosphomolybdate Keggin has not been investigated. It is only known that a dilute aqueous solution of the salt is unstable. ${ }^{1}$ Hence to address the question of the nature of the colloidal objects in an aqueous dispersion of ammonium phosphododecamolybdate Keggin, we started our investigation with a very dilute sonicated dispersion of the salt. An investigation of this sonicated aqueous colloidal dispersion reveals the spontaneous formation of small spheres $(5-50 \mathrm{~nm}$ radii) that with time generate micrometer sized "pea-pod"-like particles and finally rods (upon acidification) as shown schematically in Figure 1. The investigation was carried out using time-resolved dynamic light scattering (DLS), transmission electron microscopy (TEM), and scanning TEM (STEM) with a high-angle annular dark field detector (HAADF) for energydispersive X-ray (TEM/EDX) elemental analyses.

\section{Materials and Methods}

Synthetic Procedure. Ammonium phosphomolybdate, $\left(\mathrm{NH}_{4}\right)_{3}$ $\left[\mathrm{PMo}_{12} \mathrm{O}_{40}\right]$ (MW $\left.=1876.35 \mathrm{~g} / \mathrm{mol}\right)$, was used as obtained from Acros chemicals without further purification. The dispersion of ammonium phosphomolybdate in water with which the experiments were carried out was prepared as follows. To $25 \mathrm{mg}(14.4 \mu \mathrm{mol})$

(10) López, X.; Maestre, J.; Bo, C.; Poblet J.-M. J. Am. Chem. Soc. 2001, 123, 9571-9576.

(11) Janik, M.; Bardin, B.; Davis, R.; Neurock, M. J. Phys. Chem. B 2006 $110,4170-4178$

(12) Rocchiccioli-Deltcheff, C.; Aouissi, A.; Bettahar, M.; Launay, S.; Fournier, M. J. Catal. 1996, 164, 16-27.

(13) (a) Mizuno, N.; Misono, M. Chem. Rev. 1998, 98, 199-217. (b) Kozhevnikov, I. V. Chem. Rev. 1998, 98, 171-198.

(14) Hill, C.; Prosser-McCartha, C. M. Coord. Chem. Rev. 1995, 143, 407455.

(15) López, X.; Nieto-Draghi, C.; Bo, C.; Avalos, J.; Poblet J. -M. J. Phys. Chem. A 2005, 109, 1216-1222.

(16) (a) Müller, A.; Seraine, C. Acc. Chem. Res. 2000, 33, 2-10. (b) Müller, A.; Roy, S. Coord. Chem. Rev. 2001, 245, 153-166.

(17) Pope, M.; Varga, G., Jr. Inorg. Chem. 1966, 5, 1249-1254

(18) Baker, M.; Lyons, P.; Singer, S. J. Am. Chem. Soc. 1955, 77, 2011-2012.

(19) Validzic, I.; van Hooijdonk, G.; Oosterhout, S.; Kegel, W. Langmuir 2004, 20, 3435-3440.

(20) Kurucsev, T.; Sargeson, A.; West, B. J. Phys. Chem. 1957, 61, 15671569.

(21) For an extensive overview on oxomolybdates in aqueous solution, see Tytko, K.; Trobisch, U. In Gmelin Handbook of Inorganic Chemistry; Katscher, H., Ed.; Springer-Verlag, Berlin, 1986; Vol. B3a, Mo suppl., pp 40-272.

(22) Berzelius, J. Poggend. Ann. Phys. Chem. 1826, 6, 1206-1208. 


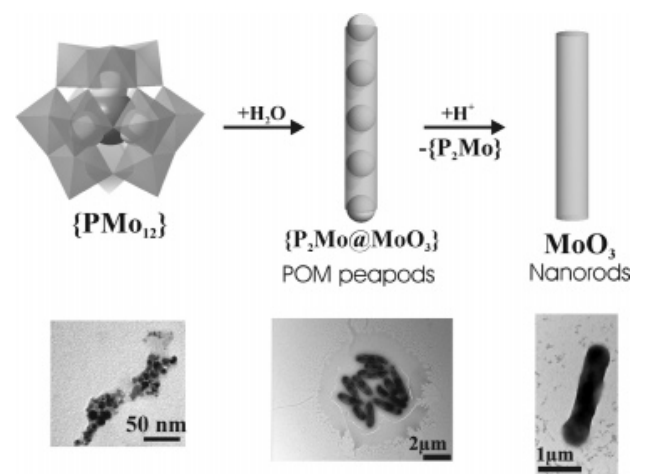

Figure 1. From spheres to rods via peapods: time-dependent stages of particle growth in an ammonium phosphomolybdate Keggin dispersion shown schematically. The corresponding bright-field TEM micrographs are shown for illustration. The $\mu_{3}$-Os of the Keggin are shown as enlarged light-gray spheres; $\mathrm{P}$ is shown as dark-gray spheres, and $\left\{\mathrm{Mo}_{3}\right\}$ caps are shown as transparent gray octahedra in a mixedpolyhedral space-filling representation of $\left\{\mathrm{PMo}_{12}\right\}$ Keggin.

of ammonium phosphomolybdate was added $20 \mathrm{~mL}$ of water. The suspension was sonicated for $10 \mathrm{~min}$, and the undissolved remnants of yellow phosphomolybdate Keggin were removed by centrifugation. The supernatant was separated and further filtered through a 3- $\mu \mathrm{m}$ (pore-diameter) solvent-resistant Millipore membrane. The $\mathrm{pH}$ of the filtrate was ca. 4.5. This filtrate was further used for analyses by DLS, TEM, and EDX/STEM. The acidification experiments to leech out the spheres from the peapods were then performed on the same filtrate after it was 3 days old. The acidification was done using $1 \mathrm{M}$ hydrochloric acid, and the $\mathrm{pH}$ of the acidified dispersion was determined to be around 2, which led to the leeching of the spheres from the peapods. (See the text). The $\mathrm{pH}$ of the dispersions for the purpose was measured with a Schott-Geräte $\mathrm{pH}$ meter.

Dynamic Light Scattering (DLS) Experiments and Analyses. DLS measurements were performed using an argon laser (Spectra Physics, series $2020, \lambda=514.5 \mathrm{~nm}$ ) operating at a laser intensity of $450 \mathrm{~mW}$ and a temperature of $298 \mathrm{~K}$. The samples were measured in Danliker cuvettes. The intensity autocorrelation functions (IACF) were obtained using a Malvern correlator. In time-resolved DLS, each measurement was performed at six angles $(30,48,66,84,102$, and $120^{\circ}$ ). At each angle, 10-20 decay curves were measured, and the decay exponent was obtained by fitting the decay curves to a second-order cumulant fit that implied the presence of more than one component in the system in addition to the starting spherical particles. The radii of the starting spherical particles obtained from the apparent translational diffusion coefficients of the DLS experiment were in the range of around 50-80 nm. However, for the peapods we analyzed the data using the model of thick rods. ${ }^{23}$ Some approximations were essential for calculating the diffusion coefficients of the peapods using their TEM dimensions. Those approximations are as follows: (1) Translational and rotational diffusion are decoupled. (2) Polydispersity in size, shape, and refractive index is neglected.

With these approximations, the diffusion coefficients of the peapods, in the limit of infinite dilution, were obtained using the following relations ${ }^{23}$

$$
\begin{aligned}
D_{0, \|} & =\frac{k_{\mathrm{B}} T\left[\log (l / d)-0.207+0.98(d / l)-0.133(d / l)^{2}\right]}{2 \pi \eta l} \\
D_{0, \perp} & =\frac{k_{\mathrm{B}} T\left[\log (l / d)+0.839+0.185(d / l)+0.233(d / l)^{2}\right]}{4 \pi \eta l}
\end{aligned}
$$

where $D_{0, \mid}$ and $D_{0, \perp}$ are the translational diffusion coefficients parallel and perpendicular to the long axis, respectively, including end effects; $k_{\mathrm{B}}$ is the Boltzmann constant; $l$ is the length and $d$ is the diameter

(23) Dhont, J. K. G. An Introduction to Dynamics of Colloids; Elsevier: Amsterdam, 1996; pp 158-160.

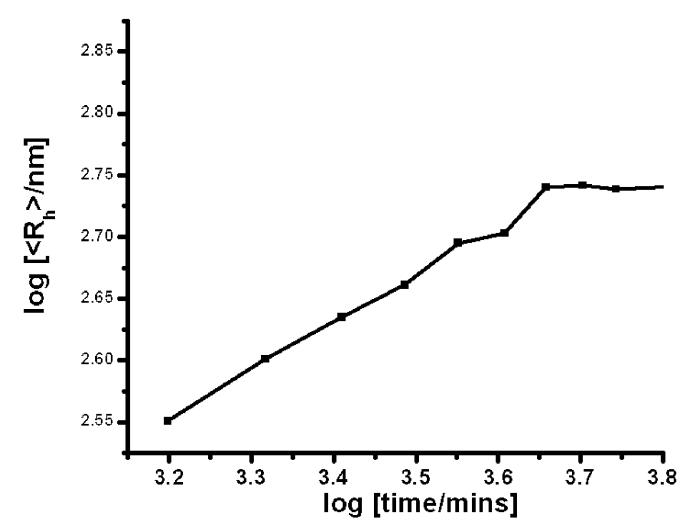

Figure 2. Power-law behavior of the time-dependent growth of particles in the Keggin dispersion. The plot shown was measured at a $66^{\circ}$ scattering angle; however, this behavior is observed for all six angles at which the experiments were carried out and is independent of the angle or the sample time used.

of the peapods; and $\eta$ is the viscosity of the water with the peapods. (Because the concentration of the Keggin used was too low, only the viscosity of water was used for calculations.) Using $l=2 \mu \mathrm{m}$, $d=500 \mathrm{~nm}$ (as obtained from TEM), $k=1.38 \times 10^{-23} \mathrm{~J} / \mathrm{K}, T=$ $298 \mathrm{~K}$, and $\eta=898 \mathrm{mPa}$ for water at $298 \mathrm{~K}$, we obtained $D_{0, \|}=$ $5.1 \times 10^{-13} \mathrm{~m}^{2} / \mathrm{s}$ and $D_{0, \perp}=2.3 \times 10^{-13} \mathrm{~m}^{2} / \mathrm{s}$. The weightedaverage translational diffusion coefficient calculated as above was $\langle D\rangle=3.3 \times 10^{-13} \mathrm{~m}^{2} / \mathrm{s}\left(\langle D\rangle=1 /{ }_{3} D_{0, \|}+2 /{ }_{3} D_{0, \perp}\right)$, whereas the experimental diffusion coefficient from DLS was $4.8 \times 10^{-13} \mathrm{~m}^{2} / \mathrm{s}$ at the smallest scattering vector $k$.

Transmission Electron Microscopy and Energy-Dispersive X-ray Analyses (EDX/STEM). Bright-field images were taken using a Tecnai 10 (FEI Company) transmission electron microscope operated at an acceleration voltage of $100 \mathrm{kV}$. Dark-field images were collected and EDX (energy-dispersive X-ray) analyses were made using a Tecnai 20 (FEI Company) transmission electron microscope operated at an acceleration voltage of $200 \mathrm{kV}$ with an EDX/STEM HAADF (scanning transmission electron microscope with a high-angle annular dark field) detector. The TEM micrographs have been processed using TIA software (Tecnai imaging and analysis software).

\section{Results and Discussion}

Our investigation was started with a very dilute sonicated aqueous dispersion of the ammonium phosphomolybdate Keggin salt using time-resolved dynamic light scattering (DLS) experiments. The apparent translational diffusion coefficients $(D)$ ascertained from the freshly prepared dispersion decreased, whereas the dynamic contrast, which is related to turbidity or the total scattering intensity, increased for a given scattering angle within 1 day. These observations clearly imply a "growth" of the particles in the colloidal dispersion of the Keggin. Indeed, the hydrodynamic radii of the particles, calculated from translational diffusion coefficients by DLS experiments (assuming spherical particles), increased from $50-500 \mathrm{~nm}$ in 2 days. The growth of the particle radius with time indicates power-law behavior (Figure 2) with an exponent close to 0.3 , pointing to Ostwald ripening, but over a limited time window of 26-66 h. At longer times, a plateau is reached, implying that particles reach a constant size. The exact morphology of the particles was determined by TEM (Figure 1).

TEM micrographs from the "fresh" dispersion showed the presence of small spherelike entities with radii in the range of 5-50 nm (Figure 1). After 2 to 3 days, the spheres start disappearing, and larger cylinders are observed, which decimates the continuous growth of the particles and results in the formation of a plateau as seen from DLS (Figure 2). These cylinders have 


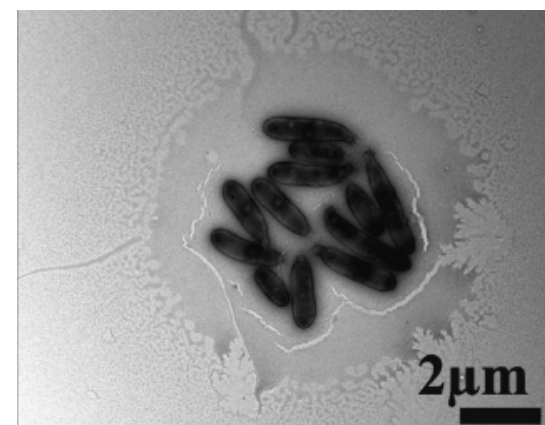

Figure 3. Bright-field TEM micrograph of a set of inorganic peapods. Note the spheres within the cylinders.

an average length of around $2 \mu \mathrm{m}$ and a diameter of $500 \mathrm{~nm}$ (Figure 3). Additionally, smaller spheres with diameters of around $200 \mathrm{~nm}$ are found to be embedded in these cylinders. The morphological resemblance of these structures to those of peapods prompted us to name them inorganic peapods. Further consistency was observed between our TEM and DLS experiments other than the formation of a plateau in the time-dependent growth profile of the particles as mentioned above.

The TEM radii of the starting spherical particles were in the range of $5 \mathrm{~nm} \lesssim R \lesssim 50 \mathrm{~nm}$, whereas the radii obtained from the apparent translational diffusion coefficients of the DLS experiment were in the range of around 50-80 nm. For the peapods, the matching of the particle dimensions from TEM and DLS was carried out. (See Materials and Methods section for a detailed analysis.)

The consistency between the calculated translational diffusion coefficient using the TEM particle dimensions $\left(3.3 \times 10^{-13} \mathrm{~m}^{2} /\right.$ s) and the experimentally obtained value $\left(4.8 \times 10^{-13} \mathrm{~m}^{2} / \mathrm{s}\right)$ from DLS implies that the time-dependent shape transformation of the particles in the Keggin dispersion from spheres to cylindrical peapods indeed represent a global picture of the dispersion and not some artifact of TEM or a statistically insignificant local phenomenon. Hence, the next imminent question is, what is the nature of these peapods?

Because of the very small concentration of Keggins, spectroscopic techniques such as ${ }^{31} \mathrm{P} \mathrm{NMR}$, attenuated total reflection infrared (ATR-IR), and Raman spectroscopy and diffraction technique such as small-angle X-ray scattering (SAXS) were of no help in answering the question. (Note that an estimate of the concentration was made by weighing the insoluble solid residue, and the concentration of the dispersion was found to be ca. 5 $\mu \mathrm{M}$.) Even selected-area electron diffraction (SAED) was not informative because the spheres and peapods turned out to be noncrystalline. We were left with semiquantitative energydispersive X-ray analyses and coupled it for confirmation with scanning transmission electron microscopy in a dark field with a high-angle annular dark-field detector (STEM-HAADF). STEM clearly showed constitutional heterogeneity in the peapods' elemental composition. The spheres in the peapods showed up as bright spots (Figure 4), implying the presence of densely packed heavier elements, whereas the cylindrical shell showed up as a darker region, implying less densely packed elements with lesser secondary electrons. EDX was coupled with STEMHAADF (high-angle annular dark field), and elemental mappings of the bright spots in STEM together with that of the darker background were carried out (Figure 4 ). The analyses consistently revealed a 2:1 $\mathrm{P} / \mathrm{Mo}$ elemental ratio in the bright spots, whereas those in the darker background did not reveal any P but only Mo. Taking into account the unreliability of the elemental estimations of lighter elements such as $\mathrm{O}$, the accuracy of its abundance by
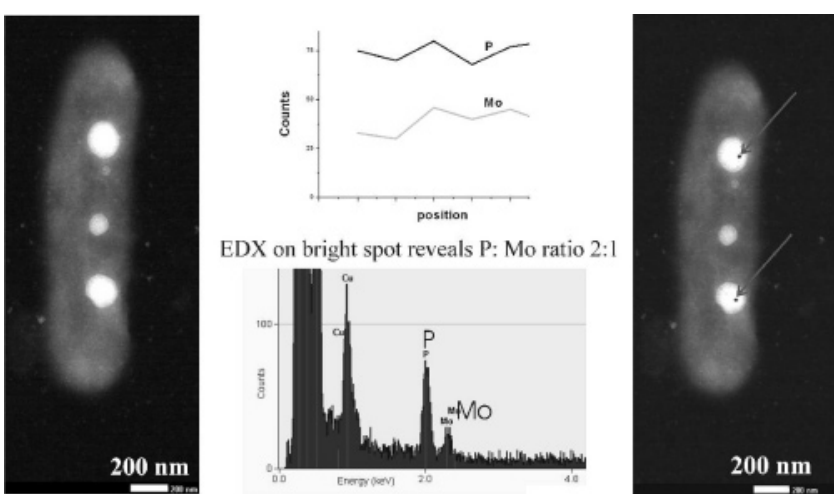

Figure 4. STEM-HAADF image of a peapod prior to (left) and after (right) EDX analysis. EDX measurements together with one representative analysis on one of the bright spots of the peapods with a 2:1 P/Mo ratio is depicted (middle). (Note that $\mathrm{Cu}$ and $\mathrm{C}$ come from the TEM grid.) The spots of beam damage in the course of EDX analyses are shown by arrows.

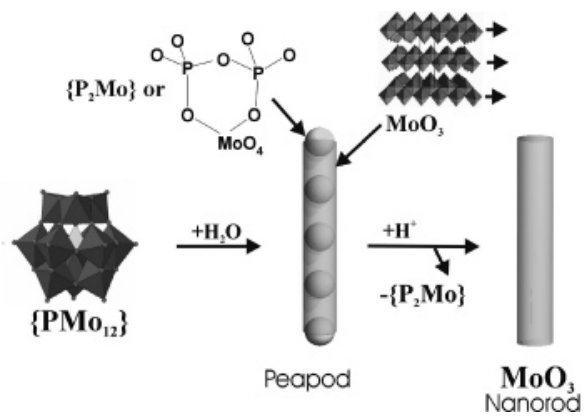

Figure 5. Overview of the whole process of peapod formation from the starting ammonium salt of $\left\{\mathrm{PMo}_{12}\right\}$ Keggin. The spheres in the peapods are proposed to be composed of $\left[\mathrm{P}_{2} \mathrm{MoO}_{11}\right]^{6-}$, whereas the "skin" is proposed to consisting of a protonated $\mathrm{MoO}_{3}$ sheet.

EDX might be doubted but not its presence. In the spheres, we observed $\mathrm{P} / \mathrm{Mo}$ in an approximate atomic ratio of $2: 1$, whereas in the cylindrical background only $\mathrm{Mo} / \mathrm{O}$ was found in a ratio $\sim 1: 10 .{ }^{24}$ On the basis of these EDX/STEM elemental analysis data, we propose $\left[\mathrm{P}_{2} \mathrm{MoO}_{11}\right]^{6-}$ (abbreviated $\left\{\mathrm{P}_{2} \mathrm{Mo}\right\}$ throughout the remainder of the letter) as the prime constituent of the spherical entities surrounded by one or more sheets ${ }^{25}$ of $\mathrm{MoO}_{3}$ of the peapods formed according to the following transformation (Figures 4 and 5):

$$
\begin{aligned}
& 2\left[\mathrm{PMo}_{12} \mathrm{O}_{40}\right]^{3-}+46 \mathrm{H}_{2} \mathrm{O} \Leftrightarrow \\
& \qquad {\left[\mathrm{P}_{2} \mathrm{MoO}_{11}\right]^{6-}+23\left[\mathrm{MoO}_{3} \cdot 2 \mathrm{H}_{2} \mathrm{O}\right] }
\end{aligned}
$$

The tendency of $\mathrm{MoO}_{3}$ to form sheets and to intercalate protons has been shown ${ }^{25}$ and is evident from their layer lattice crystal structure (Figure 5). The protons can be thought of as being intercalated between the layer lattices of $\mathrm{MoO}_{3}$ sheets. ${ }^{25}$ Even their ability to form rods has recently been explored. ${ }^{26} \mathrm{We}$ sought additional support for the plausibility of the reaction proposed above. Indeed, after acidification, the peapods lost their spherical $\left\{\mathrm{P}_{2} \mathrm{Mo}\right\}$ seeds as a result of acid-induced cleavage of one of the

(24) Note that initially formed spherical Keggin particles with a radius of around $5-50 \mathrm{~nm}$ revealed a P/Mo ratio of around $1: 12$ or less. Hence, the formation of lacunary Keggins (with one or more missing Mo cap) or even higher lacunary derivatives in those starting particles may not be unlikely, which is in line with the instability of the phosphododecamolybdate Keggins in very dilute aqueous dispersions as reported in the literature. ${ }^{\text {la }}$

(25) Adams, S.; Ehses, K.-H.; Spilker, J. Acta Crystallogr. 1993, B49, 958967.

(26) Niederberger, M.; Krumeich, F.; Muhr, H.-J.; Müller, M.; Nesper, R. J. Mater. Chem. 2001, 11, 1941-1945. 
$\mathrm{P}-\mathrm{O}-\mathrm{Mo}$ bonds, and in TEM, only $\mathrm{MoO}_{3}$ cylinders were left. However, many dark spots were found around the cylinders, which were the remaining phosphates (as ascertained by EDX/ STEM analyses). The overall phenomenon has been summarized schematically in Figure 5.

It is perhaps appropriate here to compare and contrast the nature of the morphological complexity in our system with that of the well-known Stöber synthesis of colloidal silica spheres. ${ }^{27}$ In Stöber's route, the hydrolyzed product of alkyl-silicates decomposes into silica, and subsequent condensation of silicic acid results in the formation of colloidal spheres. In our case, we have an additional level of complexity. Here we have a comparable hydrolytic decomposition process that forms two morphologies (i.e., $\left\{\mathrm{P}_{2} \mathrm{Mo}\right\}$ spheres and $\mathrm{MoO}_{3}$ plates) at the same time that interfere, ultimately leading to the formation of peapods.

An important point within this context might be worth noting. The nature of the counter ions of the phosphomolybdate Keggin plays an important role in the formation of these structures. For instance, a more soluble sodium salt of phosphomolybdate does not form peapods as discussed here; instead, they remain in the solvated state in solution.

We now speculate as to why peapods are formed. Physically, it is relatively easy to understand the formation of a cylindrical morphology rather than a sphere. This is because a cylinder, unlike a sphere, does not require the generation of $12 c_{5}$ axes and the breaking of 12 contacts thereof. The question of why spherical $\left\{\mathrm{P}_{2} \mathrm{Mo}\right\}$ forms spheres wrapped in a sheet of $\mathrm{MoO}_{3}$ remains to be answered. However, from a chemical point of view it is intriguing to see how the less-soluble ammonium salt of twocomponent phosphododecamolybdate Keggin goes back to its two starting components, $\mathrm{MoO}_{3}$ and $\mathrm{PO}_{4}{ }^{3-}$, via a series of shape transitions. Though the exact mechanism of this shape transition is not yet understood, we may allude to an architectural concept for stress analysis as an analogy. It is known that any architecture crumbles along the weaker lines of its construction when subjected to a yielding force. Perhaps likewise the phenomenon as outlined here traces out the fault lines of phosphomolybdate Keggin's molecular construction (i.e., the linking between the central tetrahedral phosphate and the four surrounding $\left\{\mathrm{Mo}_{3}\right\}$ caps) via the $\mu_{3}$-Os (Figure 1) along which the Keggin salt decomposes, forming macroscopic peapods and finally $\mathrm{MoO}_{3}$ cylinders. It might also be mentioned that we believe that stress in the molecular Keggin arises along the $\mu_{3}$-Os (the alleged fault-lines) because components on either side of the faultine (i.e., the $\mathrm{PO}_{4}$ and $\mathrm{MoO}_{3}$ caps) prefer different curvatures. Additionally, the

(27) Stöber, W.; Fink, A.; Bohn, E. J. Colloid Interface Sci. 1968, 26, 62-69.
Mo-O-Mo angular strain in the starting molecule (along the $\mu_{3}$-Os marking the fault line) is released as thermodynamically more stable $\left\{\mathrm{P}_{2} \mathrm{Mo}\right\}$ species are formed in the peapods (Figure 1). Apparently, this strain/frustration on a molecular scale leads to the thermodynamic instability of the starting material and ultimately results in the formation of a thermodynamically stable macroscopic composite (peapods). However, the stress in the molecule can become active only to disintegrate the whole structure into its components if and only if the starting POM salt is less/sparingly soluble. Because the phenomenon described here leads to the formation of new shapes (on macroscopic length scales) as a result of degeneration (on a molecular scale), we propose to name this phenomenon degenerative morphogenesis. This experimentally observed phenomenon could be exploited for facile hybrid/composite materials design. Unlike the larger clusters, the smaller and less-soluble salts of POMs, such as that of the ammonium salt of the phosphomolybdate Keggin discussed here, do not show spherical shell-like superstructures; ${ }^{2-4}$ instead, they show peapod-shaped structures as outlined here.

\section{Conclusions}

In this letter, we have described the unusual phenomenon of the spontaneous formation of micrometer-sized inorganic peapods starting from a sonicated aqueous dispersion of ammonium phosphomolybdate Keggin. The structures have been characterized by DLS, TEM, and STEM/EDX analyses. These analyses have revealed the decomposition of the starting phosphomolybdate Keggin along the lines of the formation of the peapods. Low solubility and the presence of more than one component in the starting precursor is necessary to observe such a phenomenon experimentally. This letter demonstrates that lesssoluble polyoxometalates, such as ammonium phosphomolybdate (with two components phosphate and molybdate), have the potential to form microscopic composites (e.g., peapods), as observed experimentally. Thinking along these lines, we might predict possible POM precursors that could be candidates for forming comparable macroscopic composite architectures. They are sparingly soluble mono-, di-, and trilacunary Keggin and Dawson-Wells ${ }^{1}$ salts in water or another appropriate solvent. Needless to say, each of these POMs, such as the phosphomolybdate Keggin discussed here, in addition to having more than one component in their molecular structures are also sparingly soluble in water.

Acknowledgment. This research was financially supported by NWO-CW.

LA700373H 